\title{
Alu-mediated deletion of SOX10 regulatory elements in Waardenburg syndrome type 4
}

\author{
Nadége Bondurand ${ }^{*, 1,2}$, Virginie Fouquet ${ }^{3}$, Viviane Baral ${ }^{1,2}$, Laure Lecerf ${ }^{4}$, Natalie Loundon ${ }^{5}$, \\ Michel Goossens ${ }^{1,2,6}$, Benedicte Duriez ${ }^{7}$, Philippe Labrune ${ }^{8,9}$ and Veronique Pingault ${ }^{1,2,6}$
}

\begin{abstract}
Waardenburg syndrome type 4 (WS4) is a rare neural crest disorder defined by the combination of Waardenburg syndrome (sensorineural hearing loss and pigmentation defects) and Hirschsprung disease (intestinal aganglionosis). Three genes are known to be involved in this syndrome, that is, EDN3 (endothelin-3), EDNRB (endothelin receptor type B), and SOX10. However, 15-35\% of WS4 remains unexplained at the molecular level, suggesting that other genes could be involved and/or that mutations within known genes may have escaped previous screenings. Here, we searched for deletions within recently identified SOX10 regulatory sequences and describe the first characterization of a WS4 patient presenting with a large deletion encompassing three of these enhancers. Analysis of the breakpoint region suggests a complex rearrangement involving three Alu sequences that could be mediated by a FosTes/MMBIR replication mechanism. Taken together with recent reports, our results demonstrate that the disruption of highly conserved non-coding elements located within or at a long distance from the coding sequences of key genes can result in several neurocristopathies. This opens up new routes to the molecular dissection of neural crest disorders.
\end{abstract}

European Journal of Human Genetics (2012) 20, 990-994; doi:10.1038/ejhg.2012.29; published online 29 February 2012

Keywords: alu elements; regulatory elements; SOX10; Hirschsprung; Waardenburg

\section{INTRODUCTION}

Waardenburg syndrome type 4 (WS4), also referred to as ShahWaardenburg syndrome or Waardenburg-Hirschsprung disease, is a neural crest disorder characterized by the association of Waardenburg syndrome features (WS, depigmentation defects and deafness) with the absence of enteric ganglia in the distal part of the intestine (Hirschsprung disease, HD). ${ }^{1,2}$ Approximately, 20-30\% of cases are due to homozygous or heterozygous mutations within the EDN3 (endothelin-3) or the EDNRB (endothelin receptor type B) genes, whereas approximately $45-55 \%$ result from heterozygous mutations within the gene encoding the SOX10 (Sry bOX10) transcription factor. ${ }^{2}$ Several of the known SOX10 mutations are responsible for extended phenotypes, including peripheral and central neurological defects, and they are referred to as PCWH (peripheral demyelinating neuropathy Central dysmyelinating leucodystrophy - WS - HD). ${ }^{3}$ However, $15-35 \%$ of WS4 cases remain unexplained at the molecular level, suggesting that other genes could be involved and/or that mutations within the known genes or their regulatory sequences have escaped previous screenings.

Recent reports show that the disruption of highly conserved noncoding elements located within or at a long distance from the coding sequences of key genes can result in several neurocristopathies, particularly HD. ${ }^{4,5}$ Thus, identifying genomic alterations that affect the conserved sequences of WS4 genes might provide further insights into the molecular aetiology of this syndrome.
Recently, sequence comparison analysis has led to the identification of several evolutionarily conserved regions with putative enhancer activity around the SOX10 locus. ${ }^{6-10}$ In particular, we and others identified five of these regulatory elements upstream of the human SOX10 gene (U1-5; U1 is localised $55 \mathrm{~kb}$ upstream of the SOX10 gene) and one downstream (D6+7) (Werner et al ${ }^{9}$; Yokota et al ${ }^{10}$ and our unpublished results). The functional relevance of each of these elements was studied in different cell lines and in zebrafish, chicken and mouse models. Most of them direct expression in several neural crest derivatives, the enteric nervous system, and melanocytes in particular. ${ }^{7,9}$ Here, we used semi-quantitative fluorescent multiplex PCR (QMF-PCR) to search for deletions within these elements and describe a WS4 patient presenting with a deletion encompassing three of them.

\section{METHODS}

A total of 13 patients presenting with a classical form of WS4, and 9 patients with its neurological variant (PCWH) were screened in this study. They were previously found negative for gene rearrangement and point mutation in the coding exons of SOX10 (WS4 and PCWH patients), as well as EDN3 and $E D N R B$ (WS4 only). DNA samples were obtained for genetic testing with informed consent, according to French law. Genomic DNA was extracted from peripheral blood leucocytes using standard protocols.

QMF-PCR protocol and primers used are available within Supplementary data (Supplementary Table 1).

${ }^{1}$ INSERM, U955, Equipe11, Hôpital Henri Mondor, Créteil, France; ${ }^{2}$ Université Paris Est, Faculté de Médecine, Créteil, France; ${ }^{3}$ AP-HP, Hôpital Bicêtre, Service de Chirurgie Pédiatrique, 94275 le Kremlin Bicêtre cedex, France; ${ }^{4}$ INSERM, U955, Plateforme de Génomique, Créteil, France; 5 INSERM, Unité U587, ORL, AP-HP, Hopital Armand Trousseau, Paris, France; ${ }^{6}$ AP-HP, Hôpital H. Mondor - A. Chenevier, Service de Biochimie et Génétique, Créteil, France; ${ }^{7}$ Institut Jacques Monod, UMR7592 CNRS, Université Paris Diderot-Paris 7, Paris, France; ${ }^{8}$ AP-HP, Hôpital Antoine Béclère, service de pédiatrie, Clamart cedex, France; 9 Université Paris Sud, UFR Kremlin Bicêtre, Le Kremlin Bicêtre cedex, France

${ }^{*}$ Correspondence: Dr N Bondurand. INSERM U955, Equipe 11, Hôpital Henri Mondor, 51 Avenue du Maréchal de Lattre de Tassigny, 94010, Créteil, France. Tel: +33149812854; Fax: +33148993345; E-mail: nadege.bondurand@inserm.fr

Received 15 November 2011; revised 10 January 2012; accepted 26 January 2012; published online 29 February 2012 
The deletion breakpoint region was amplified by long range PCR using the Expand Long Template PCR system (Roche Diagnotics, Mannheim, Germany) and sequenced using the following primers: $5^{\prime}$-CCTTCCGCAAAGACTTGCT G- $3^{\prime}$ and $5^{\prime}$-ATGGAACCTGGGAAGACACC- $3^{\prime}$. Alu1 and Alu2 were amplified and sequenced in both parents using the following primers: $5^{\prime}$-ATG GAACCTGGGAAGACACCT- $3^{\prime}$ and 5'-AAGCACAATCGGCTAAGAGG-3'; and 5'-ACCCAGTTTGGAGTGCAGTG-3' and 5'-CCTTCCGCAAAGACTTGCTG-3'.

Array CGH analysis was performed using the Agilent Human G3 CGH Microarray 4x180K Kit (Agilent Technologies, Massy, France) and the data were analysed with an Agilent scanner (G2565CA) and the Feature Extraction program (V10.7.3.1, Agilent technologies). Copy number variations were considered significant if defined by three or more contiguous oligonucleotides.

Paternity was verified by the use of several microsatellites from different chromosomes (the linkage-mapping set, Applied Biosystem, Carlsbad, CA, USA)

\section{RESULTS AND DISCUSSION}

QMF-PCR is known to be a sensitive method for the detection of SOX10 gene dosage anomalies. ${ }^{11}$ The same strategy was used to screen for deletions within six regulatory sequences of interest (U1-5 and D6+7 elements, Figure 1a) in WS4 and PCWH patients with no molecular explanation. We identified a heterozygous deletion encompassing regulatory elements U1, 2, and 3 (Figure 1a, see regions indicated by black arrowheads) in a patient presenting with a classical WS4 phenotype.

The propositus was born at term after an uneventful pregnancy. Both healthy parents originated from the Congo. The mother already had two children, a healthy boy and a baby boy who died of an unknown cause at 1 year of age. The patient presented with major abdominal distension at birth, and he did not pass meconium. X-ray showed a dilatation of the ileum and right colon, and a clear change in calibre between the right and transverse colon. A colostomy was performed one day after birth, which was followed by ileo-rectal anastomosis using the Duhamel procedure after 3 months. Histology revealed the absence of ganglionic cells extending up to the right colon region, followed by a transition zone in the last $2 \mathrm{~cm}$. The postoperative course was complicated by volvulus and occlusion, leading to a secondary ileostomy intervention and Hartman operation. Ileo-anal anastomosis was performed at 18 months of age and the associated lateral ileostomy was closed 6 months later. The patient suffered numerous episodes of acute gastroenteritis up to the age of four-and-a-half, necessitating frequent hospitalisation. He also experienced episodes of microcytic anaemia. He is now a 5 -year-old boy with a normal growth curve. Neurological examination was normal.

WS was diagnosed at the age of 3 months based on the suspicion of deafness, bright blue irides, and a small white frontal forelock. A severe to profound bilateral deafness was confirmed at 8 months. MRI and a temporal bone CT scan revealed no abnormality. He was fitted with hearing aids at 21 months and showed improvement on a few frequencies using the right ear. Left ear cochlear implantation was proposed at the age of 4 . The hearing thresholds with cochlear implantation reached $25 \mathrm{~dB}$ at all frequencies 18 months later. Speech recognition was assessed for simple sentences and he now expressed through isolated word and gesture.

Analysis of DNA samples from both parents revealed de novo occurrence of the deletion. To confirm the deletion by a second method and further delineate its extent, we next performed CGHarray in the patient and parents. This analysis revealed only one de novo copy number variation that encompassed five probes localised in the distant $5^{\prime}$ region of SOX10 on chromosome 22 (Figure 1b),

a
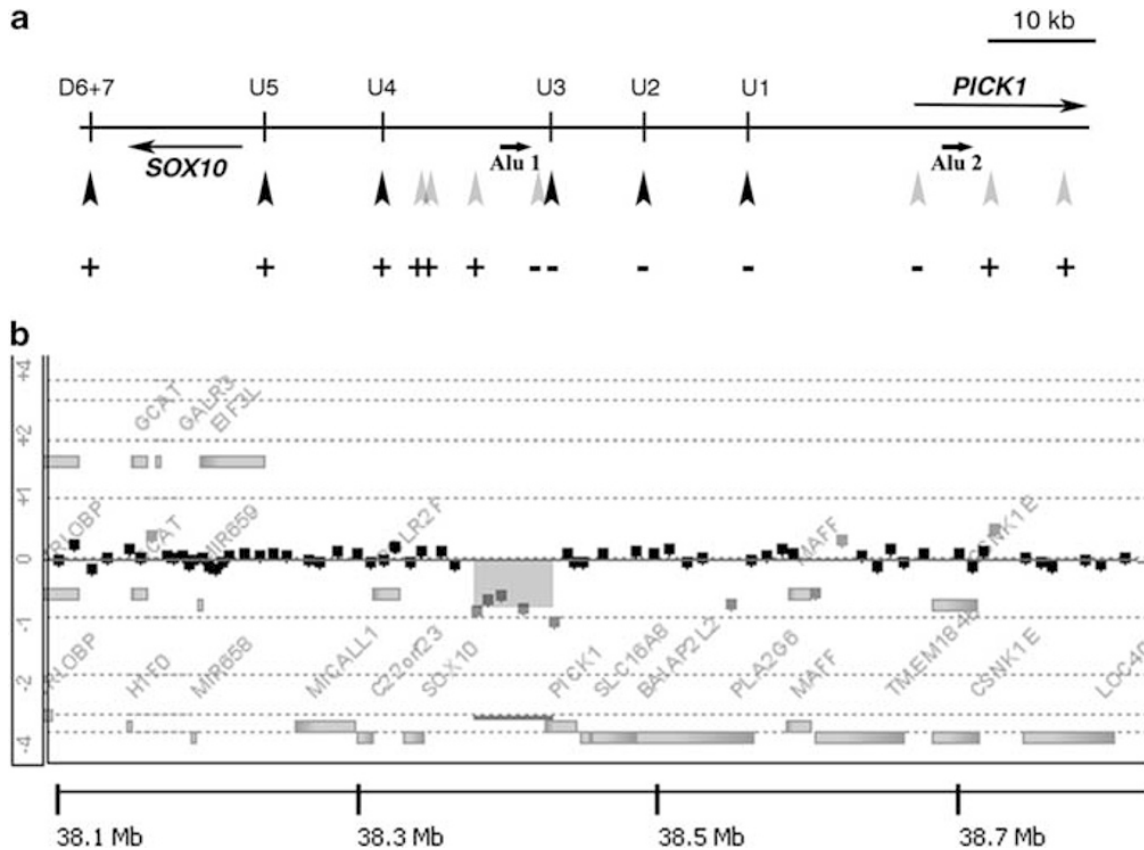

Figure 1 Characterisation of SOX10 regulatory sequences deletion identified by QMF-PCR and CGH array. (a) Schematic representation from centromere to telomere of the deletion identified by QMF-PCR. The scheme indicates the orientation of the SOX1O and PICK1 genes, with the localisation of the enhancers (U1-5 and D6+7). Segments analysed by the initial QMF-PCR are indicated by black arrowheads, whereas additional primers used to delineate the deleted regions are indicated by grey arrowheads (see Supplementary Table 1 for primer sequences). QMF-PCR results are shown below: ' + '=not deleted or '-'=deleted. Localisation of Alu1 and 2 sequences described in Figure 2 are indicated. (b) Graphical overview of the CGH-array results encompassing the chromosome 22 deletion. The results are represented using the Genomic Workbench software (V5.0.14, Agilent technologies). Dots show the relative intensities as deviation from the horizontal line of $\log 2$ Ratio $=0$. The deleted region is highlighted. The genes are represented and the genomic locations are indicated in the horizontal scale. 
confirming the results obtained using QMF-PCR. Analysis of the deletion extent identified by both techniques mapped the proximal limit between U3 and U4 sequences and the distal limit within the PICK1 gene. On the basis of these results, we chose additional sets of primers localised between regulatory sequences $\mathrm{U} 3$ and $\mathrm{U} 4$, as well as within the PICK1 gene (Figure 1a, grey arrowheads). We thus mapped the centromeric border of the deletion to a region located $31-24 \mathrm{~kb}$ upstream of the SOX10 start codon, and its telomeric border between exons 1 and 4 of the PICK1 gene.

The deletion breakpoint was then amplified by long range PCR and sequenced. Comparison of the patient (Figure 2a) and surrounding reference sequences revealed that the deletion occurred between two highly similar sequences, which were identified as Alu repeats using Repeat Masker (http://www.repeatmasker.org/). An alignment of the propositus' mutant sequence and these two Alu sequences (referred to as Alu1 for the sequence located near U3 and Alu2 for the PICK1 intronic Alu sequence) is shown in Figure 2b. These Alu sequences belonged to different subfamilies, that is AluSx for Alu1, and AluSp for Alu2. However, careful analysis of the sequence located in the central part of the patient' recombined allele revealed $38 \mathrm{bp}$ that did not perfectly match any of the two Alu sequences (Figures 2a and b, boxed regions). Sequencing of Alu1 and Alu2 from both parents established

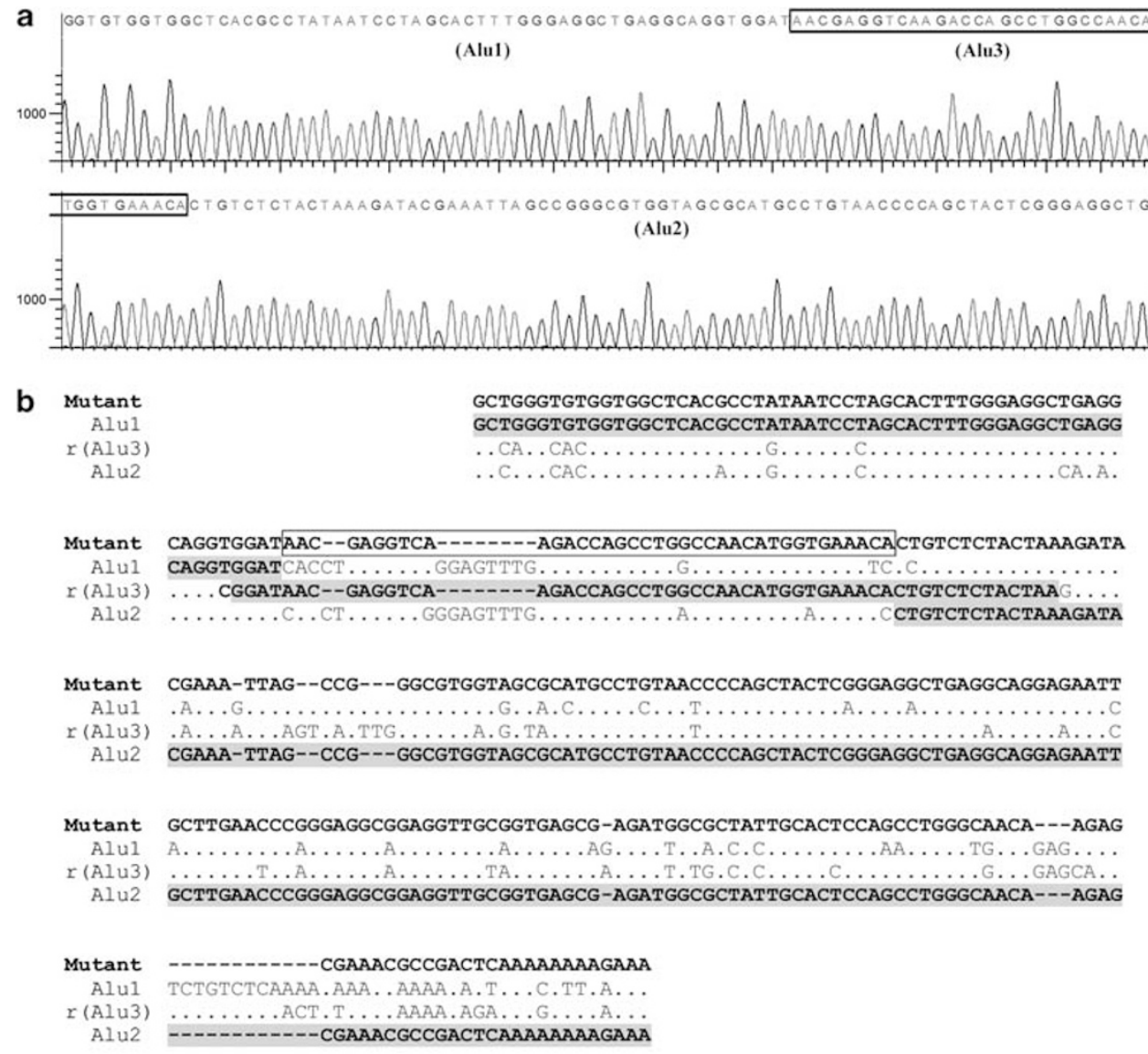

C

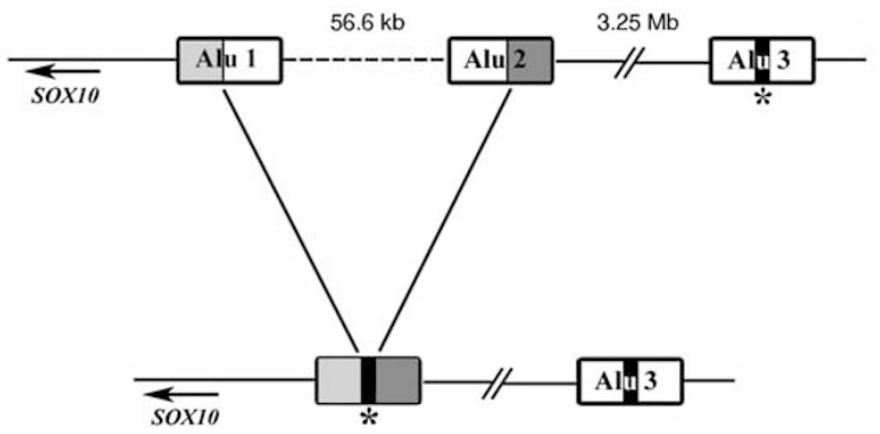

Figure 2 Sequence analysis of the breakpoint region (a) Chromatogram of the junction fragment corresponding to the mutant allele amplified from the propositus (b) Alignment of mutant and Alu1, 2 and 3 sequences. The mutant sequence observed in the propositus is shown on the first line. Dots indicate conserved nucleotides. Non-conserved nucleotides are indicated in normal upper case. The patient's mutant allele and the respective Alu1, 2, and 3 sequence fragments maintained during the deletion event are indicated in bold and highlighted in grey. The $38 \mathrm{bp}$ region homologous to part of the Alu3 sequence is boxed in a and b. (c) Top: schematic representation of Alu 1, 2, and 3 from centromere to telomere. Bottom: deletion detected in the propositus. Fragments of Alu1 and 2 maintained during the deletion event are indicated in grey. The 38-bp insertion corresponding to the Alu3 sequence (Genbank accession number NT_011520.12) is indicated in black (and *). 
that these variations were not inherited polymorphisms, but rather suggested the occurrence of a complex rearrangement mechanism. Attention was therefore focused on this $38 \mathrm{bp}$ region. BLAST analysis revealed its $100 \%$ identity with a third Alu sequence (referred as Alu3) that uniquely occurs in the human genome. Alignment presented in Figure $2 \mathrm{~b}$ also includes Alu3 sequence, highlighting the perfect identity with the $38 \mathrm{pb}$ boxed region. Alu3 belonged to a third subfamily, AluSg, and was located on chromosome 22 at a distance of $3.25 \mathrm{Mb}$ on the telomeric side of Alu2 (Figure 2c). Overall, these observations clearly suggested that a complex rearrangement occurred between the three Alu sequences, leading to the deletion of $56.5 \mathrm{~kb}$ localised between Alu1 and 2 (including exons 1, 2, and 3 of PICK1 and the $\mathrm{U} 1, \mathrm{U} 2$, and U3 SOX10 enhancers) as well as the insertion of $38 \mathrm{bp}$ of the Alu3 sequence (Figure 2c). CGH experiments failed to detect any more complex rearrangement affecting chromosome 22.

Such an event could be mediated by different mechanisms, including recombination or replication-based mechanisms. ${ }^{12,13}$ Of these, the FoSTeS/MMBIR model (fork stalling and template switching/ microhomology-mediated break-induced replication, FoSTeS/ MMBIR) is often proposed when the complexity of the rearrangement cannot be explained using classic recombination. ${ }^{13}$ According to this model, the DNA replication fork stalls at a specific position during replication, at which point the lagging strand disengages from the original template, before it transfers and anneals to another replication fork by virtue of microhomology at the $3^{\prime}$ end, primes and restarts DNA synthesis. This series of events can occur several times. The rearrangement characterized in the current study could be explained by a two-step FoSTeS/MMBIR mechanism mediated via the $4 \mathrm{bp}$ and $13 \mathrm{bp}$ microhomology found at the Alu1/Alu3 and Alu3/Alu2 breakpoints (see sequences in Figure $2 \mathrm{~b}$ and model in Figure 3).

The clinical outcome was in agreement with the known functions of the deleted sequences. A transgenic mouse analysis demonstrated the importance of $\mathrm{U} 1$ and $\mathrm{U} 3$ sequences during enteric nervous system development. ${ }^{7,9}$ Disparate results were obtained in relation to their expression profile in melanocytes. ${ }^{7,9,10}$ Nevertheless, the crucial function of $\mathrm{U} 1$ during this process was highlighted by the description of a $16 \mathrm{~kb}$ deletion encompassing this regulatory element in the Sox $10^{\mathrm{Hry}}$ mouse model, which is characterized by distal intestinal aganglionosis and severe hypopigmentation. ${ }^{6}$

The deletion described here also encompassed the U2 regulatory element, which is known to be active in oligodendrocytes. However, this element is only partly responsible for the Sox 10 expression pattern in this lineage, ${ }^{14}$ making the absence of neurological defects observed in the patient consistent with our findings. Finally, the rearrangement also led to a partial heterozygous deletion of PICK1 (protein inter-

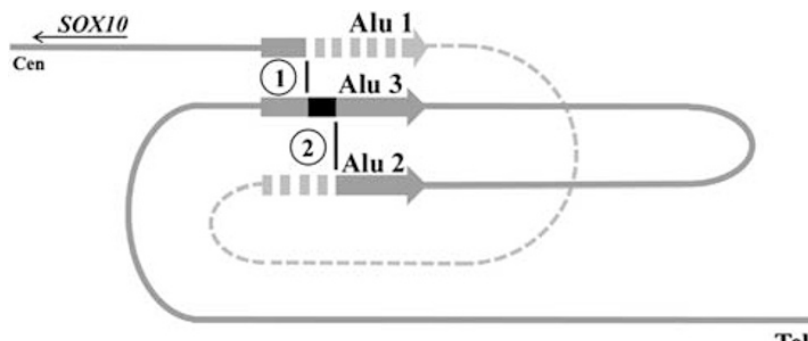

Tel

Figure 3 Proposed model to explain the rearrangement observed. Schematic representation from centromere to telomere of the region analysed. Respective orientations of SOX1O, and Alu1, 2, and 3 sequences are indicated by arrows. Numbers 1 and 2 indicate the localisation of proposed FoSTeS/MMBIR events. Doted lines indicate deleted regions. acting with $\mathrm{C}$ kinase 1 ), encoding a peripheral membrane protein implicated in the trafficking of multiple proteins. ${ }^{15}$ Mouse model studies have demonstrated its crucial function in acrosome formation and synaptic plasticity. ${ }^{15,16}$ Homozygous missense mutations were identified in patients presenting with globozoospermia, ${ }^{17}$ whereas polymorphisms were associated with schizophrenia in some populations but not all. ${ }^{18}$ It seems unlikely that the heterozygous partial deletion of PICK1 contributes to the WS4 phenotype.

$A l u$ repeats are proposed to have a number of functions in the human genome and they certainly have a major impact on the genomic architecture and are known to regulate gene expression. They are believed to contribute to about $0.4 \%$ of all human genetic diseases either via insertion events or inter-/intra-chromosomal homologous recombination, an event that can occur at very high frequencies in particular diseases. ${ }^{19-21}$ The current study identified a novel Alu-mediated deletion that resulted in WS4. Screening of SOX10 regulatory sequences should therefore be considered in molecular diagnostic of WS4, although deletion frequency (one identified among 22 patients) does not justify its use in first intention procedures. Apart from diagnosis implications, our results strongly suggest that $\mathrm{U} 1$ and $\mathrm{U} 3$ enhancers are of major importance in the physiological regulation of SOX10 expression in human.

\section{CONFLICT OF INTEREST}

The authors declare no conflicts of interest.

\section{ACKNOWLEDGEMENTS}

This work was supported by INSERM and Agence Nationale de la Recherche ANR-JCJC-2010 to NB. We would like to thank Bruno Costes and Natacha Martin for sequencing, Valerie Delattre and Wilfried Verbecq-Morlot for CGH experiments.

1 Amiel J, Sproat-Emison E, Garcia-Barcelo $\mathrm{M}$ et al: Hirschsprung disease, associated syndromes and genetics: a review. J Med Genet 2008; 45: 1-14.

2 Pingault V, Ente D, Dastot-Le Moal F, Goossens M, Marlin S, Bondurand N: Review and update of mutations causing Waardenburg syndrome. Hum Mutat 2010; 31: 391-406.

3 Inoue K, Khajavi M, Ohyama T et al: Molecular mechanism for distinct neurological phenotypes conveyed by allelic truncating mutations. Nat Genet 2004; 36: 361-369.

4 Amiel J, Benko S, Gordon CT, Lyonnet S: Disruption of long-distance highly conserved noncoding elements in neurocristopathies. Ann NY Acad Sci 2010; 1214: 34-46.

5 Emison ES, McCallion AS, Kashuk CS et al: A common sex-dependent mutation in a RET enhancer underlies Hirschsprung disease risk. Nature 2005; 434: 857-863.

6 Antonellis A, Bennett WR, Menheniott TR et al: Deletion of long-range sequences at Sox 10 compromises developmental expression in a mouse model of Waardenburg-Shah (WS4) syndrome. Hum Mol Genet 2006; 15: 259-271.

7 Antonellis A, Huynh JL, Lee-Lin SQ et al: Identification of neural crest and glial enhancers at the mouse Sox 10 locus through transgenesis in zebrafish. PLoS Genet 2008; 4: e1000174.

8 Betancur P, Bronner-Fraser M, Sauka-Spengler T: Genomic code for Sox10 activation reveals a key regulatory enhancer for cranial neural crest. Proc Natl Acad Sci USA 2010; 107: 3570-3575.

9 Werner T, Hammer A, Wahlbuhl M, BosI MR, Wegner M: Multiple conserved regulatory elements with overlapping functions determine Sox10 expression in mouse embryogenesis. Nucleic Acids Res 2007; 35: 6526-6538.

10 Yokota Y, Saito D, Tadokoro R, Takahashi Y: Genomically integrated transgenes are stably and conditionally expressed in neural crest cell-specific lineages. Dev Biol 2011; 353: 382-395.

11 Bondurand N, Dastot-Le Moal F, Stanchina L et al: Deletions at the SOX10 gene locus cause Waardenburg syndrome types 2 and 4. Am J Hum Genet 2007; 81: $1169-1185$.

12 Gu W, Zhang F, Lupski JR: Mechanisms for human genomic rearrangements. Pathogenetics 2008; 1: 4

13 Zhang F, Khajavi M, Connolly AM, Towne CF, Batish SD, Lupski JR: The DNA replication FoSTeS/MMBIR mechanism can generate genomic, genic and exonic complex rearrangements in humans. Nat Genet 2009; 41: 849-853.

14 Kuspert M, Hammer A, Bosl MR, Wegner M: Olig2 regulates Sox10 expression in oligodendrocyte precursors through an evolutionary conserved distal enhancer. Nucleic Acids Res 2011; 39: 1280-1293.

15 Xu J, Xia J: Structure and function of PICK1. Neurosignals 2006; 15: 190-201. 
16 Xiao N, Kam C, Shen C et al: PICK1 deficiency causes male infertility in mice by disrupting acrosome formation. J Clin Invest 2009; 119: 802-812.

17 Liu G, Shi QW, Lu GX: A newly discovered mutation in PICK1 in a human with globozoospermia. Asian J Androl 2010; 12: 556-560.

18 Ishiguro $H$, Koga M, Horiuchi $Y$ et al: PICK1 is not a susceptibility gene for schizophrenia in a Japanese population: association study in a large case-control population. Neurosci Res 2007; 58: 145-148.
19 Deininger PL, Batzer MA: Alu repeats and human disease. Mol Genet Metab 1999; 67: 183-193.

20 Franke G, Bausch B, Hoffmann MM et al: Alu-Alu recombination underlies the vast majority of large VHL germline deletions: molecular characterization and genotypephenotype correlations in VHL patients. Hum Mutat 2009; 30: 776-786.

21 Hasler J, Strub K: Alu elements as regulators of gene expression. Nucleic Acids Res 2006; 34: 5491-5497.

Supplementary Information accompanies the paper on European Journal of Human Genetics website (http://www.nature.com/ejhg) 